\title{
Plant Disease Detection Using Convolutional Neural Networks
}

\author{
Vempaty Prashanthi, Kanakala Srinivas \\ ${ }^{1}$ Gokaraju Rangaraju Institute of Engineering and Technology, India, prashuvempaty@gmail.com \\ ${ }^{2}$ VNR Vignana Jyothi Institute of Engineering and Technology, India, srinivaskanakala@ gmail.com
}

\begin{abstract}
Detection of various plant infections is the essential task for avoiding the losses in harvest and amount of the farming product. The research of the plant infections involves the study of graphical detectable patterns visible on the plant. Wellbeing examining and infection finding on plants is extremely crucial for environmental agriculture. It is extremely tough to examine the plant disease physically. It needs enormous quantity of work, skills in the plant infections, and also need the extreme execution time. Therefore, image processing is applied for the discovery of plant diseases. This detection includes the phases like image acquisition, image pre-processing, image segmentation, feature extraction and classification. This paper discusses the techniques required used for the discovery of plant diseases utilizing the leaves images. This paper also examined some segmentation and feature extraction algorithm utilized in the plant diseased detection.
\end{abstract}

Key words : Neural networks, disease detection, leaf images

\section{INTRODUCTION}

The issue of productive plant ailment assurance is firmly identified with the issues of manageable horticulture and environmental shift [1]. Research outcomes show that environmental transformation can adjust phases and paces of pathogen improvement; this can likewise alter have opposition, which prompts physiological changes of host-pathogen communications [2,3]. The circumstance is additionally confused with the way that, these days, maladies are moved all inclusive more effectively than any other time in recent memory. New maladies can happen in locations wherever they were beforehand anonymous and, characteristically, everywhere there is no nearby aptitude to battle them [4-6]. Unpracticed insecticide use will affect the advancement of long haul obstruction of the pathogens, seriously diminishing the capacity to retaliate. Convenient and exact determination of plant illnesses is a few of the mainstays of exactness horticulture [7]. So as to accomplish precise plant sickness diagnostics a plant pathologist ought to have great perception abilities with the goal that one can distinguish trademark side effects [8]. Abusing basic computerized picture preparing methods, for example, shading investigation and maximum level [9] were utilized along with the point of identification and characterization of plant ailments. Different methodologies are presently utilized for distinguishing plant maladies and many normal are fake neural systems (ANNs) [10] and Support Vector Machines (SVMs) [11]. These are joined with various techniques for picture preprocessing for better element extraction. The technique portrayed right now an alternative approach in recognizing plant infections employing the philosophical convolutional neural system planned and tweaked in order to suit precisely to the databank related to plant's leaves which is assembled autonomously for differing plant sicknesses. The development and oddity of the created paradigm lie down in its effortlessness; solid leaves and foundation pictures are in accordance with different styles, empowering the model to recognize ailing leaves and sound ones or from the earth by utilizing profound CNN. Perceive and group maize plant malady a computerized framework has been executed utilizing calculation, for example, chain code networks jumping box strategy and minute examination. To quantify seriousness of Rust malady on maize, ailment spot has divided to discover spot edge and plant sickness seriousness has estimated by computing the remainder of infection spot zone and leaf zone. At long last, we will finish up with a couple of plans on the most proficient method to improve the degree and consequences of the venture and how it will support the individuals and market in giving ailment discovery."

The remainder paper is sorted out as: Section 2 discuses related work, Section 3 contains strategy, Section 4 discuses accomplished outcomes and related conversation, lastly, Section 5 conclusions.

\section{LITERATURE SURVEY}

Executing the suitable administration procedures such as fungicide products, illness explicit substance applications, and direction management over insecticide requests might prompt quick data on harvest wellbeing and infection discovery. This might encourage the management of sicknesses and increase efficiency. In [12], writers presented, 
survey, and perceive the interest towards building up a fast, financially savvy, and dependable wellbeing observing sensor which encourages progressions in agribusiness. After investigation of their work and examination displayed by the authors of [13-16], it was chosen to utilize picture handling sickness acknowledgment approach among different methodologies generally utilized for plant malady diagnostics, for occurrence, dual-beached ribonucleic corrosive (RNA) investigation, nucleic corrosive tests, and microscopy. In [17], the authors have exhibited a review of notable traditional strategies for include extraction. Because of the fast advancement of Artificial Intelligence (AI), research right now centered around applying these methodologies and methods. The researchers in [18-21] introduced the profound knowledge strategies for explaining much complicated undertakings in various territories of study in science, bioinformatics, biomedicine, mechanical autonomy, and 3D advances. The detection of plant diseases can be done using the network coding scheme [22-25]. Different data stream techniques, ANN and data analytics are also some of the techniques [26-29] which can be used for detection. CNN techniques are used for diabetics and cancer detection [30, 31]. In our examination, we abuse the profound knowledge strategy for plant ailment acknowledgment, motivated by emerging of profound knowledge systems and their usage. Broad hunt of the cutting edge writing yielded no proof that specialists investigated profound learning style of plant maladies acknowledgment from the leaf pictures. Our technique for acknowledgment by applying profound CNN is introduced in segments beneath. Right now, talk about the accumulated information and its utilization right now how the leaves are arranged.

\subsection{Data Gathering}

The computer vision used in this project mainly depends on the images of the leaves. So, we decided to capture few images of leaves and utilize them to make a model facilitates in discovery of plant disease. The images are caught manually with a white background using a camera of 13MP. Each image contains only one leaf. A total of about 600 images of leaves are captured which are used for training and validating the model. A total of 150 images are used for testing the model which was built using the training data.Two main diseases of the maize leaves namely Common rust and Cercospora Figure 1 are some of the leaf images used for detection.

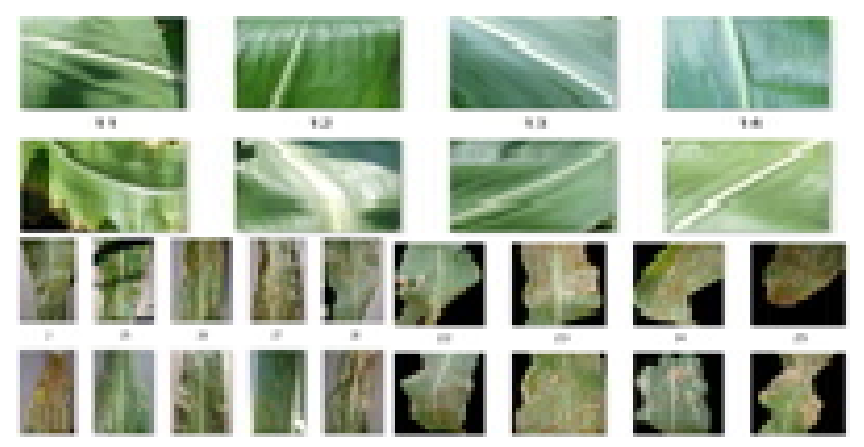

Figure. 1 : Images of leaves.
All the images that are captured are utilized in building/training a model through convolutional neural network that helps in identifying the disease. The quality of plant leaves is being assessed manually by people at various stages. This approach is error prone, time consuming and difficult.

To overcome these drawbacks, the process of detection of plant disease must be done using some computer/machine-based model. With this model, the process of disease detection may be done with in short amount of time and with great ease.

\section{PROPOSED SYSTEM}

A computer vision-based ailment discovery System to evaluate the illness of maize plant is proposed to beat the downsides of the current framework.

The Disease Detection System utilizes a prepared model to identify the ailment of the maize plant. The model is prepared by utilizing recently marked (Common Rust, Cercospora, no malady) pictures of maize leaves with the assistance of AI calculations like convolutional neural systems. After a model is prepared it is approved for preparing precision and is trailed by testing of the model with various arrangement of pictures both for approving and testing of the model. At that point the model is incorporated to the UI, right now, permit the client to identify the maize plant illness.

\subsection{Modules of System}

The modules that are present in Disease Detection System and their roles are:

\section{Plant leaf handling module}

The Plant leaf handling system contains the following: Camera and computer. The camera captures the images and uploads to the computer which acts as a server. The computer then stores the images. These images are assessed using the disease detection module.

\section{Disease Detection module}

The Disease Detection module consists of the following operations: Pre-process, Feature Extraction and selection, Classification, Return results. Figure. 2 depicts disease detection system.

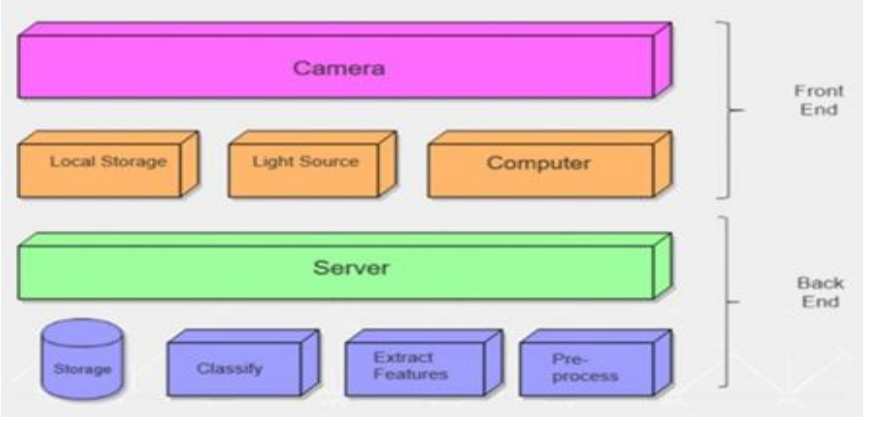

Figure 2: System Architecture of Disease Detection System 
The Disease Detection module assesses the images. It pre-processes the images, then extracts the features from the images like colour, shape etc. using neural networks. Using the extracted data, it then classifies the plant leaves as healthy or disease affected. Then the results are sent back to the plant leaf handling system where they are shown to the user.

\section{IMPLEMENTATION AND EXPERIMENTATION}

The implementation of the Disease Detection System involves the following:

1. Environment: The Disease Detection System utilizes python as its underlying programming language along with many other python dependencies. To properly manage all the dependencies of the python language, Anaconda navigator software is installed. The creation of the environment and installing all the software along with required dependencies such as NumPy, Matplotlib, Keras, TensorFlow, PIL (Python Imaging Library)

2. Pre-processing: The captured images from the camera to the Disease Detection System looks like the images in the Figure 3 shown below

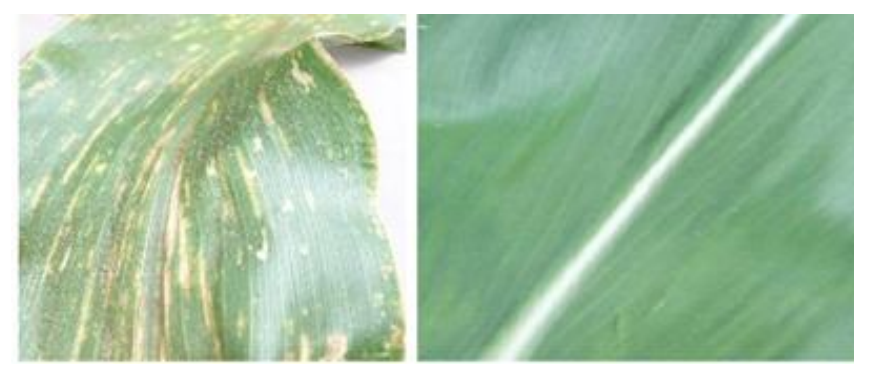

Figure 3: Images of leaves before pre-processing

As the Disease Detection system assess the leaves, the excess background image can be cropped for faster processing of the image. The captured images might look like as shown in the Figure 4.

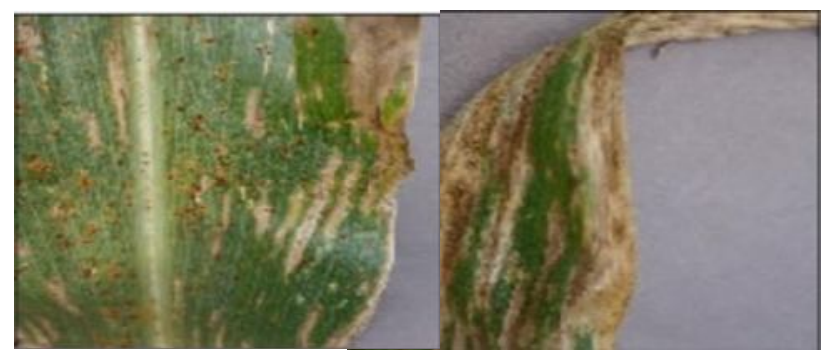

Figure 4: Images of Leaves after croping

These cropped images are fitted into a graph of size $250 \times 200$ i.e., the images are divided into blocks that can be used in training the model. But for understanding purpose, the images are shown as they are in fig 6.2 along with an axis which helps in understanding us, that the image is divided into $250 \times 200$ blocks. The pre-processed images as shown in the figure are labelled as 0.0 or 1.0 for good and bad respectively. The labelled images are as shown in the figure 5. The final output for the pre-processing images are the labelled images of the leaves of dimensions 250 X 200. These dimensions fit exactly as the input dimensions for the neural network. But for better results, the dimensions of the images can be increased, which in-turn require higher computational resources and time required to train the model. Hence, for this project the dimensions of $250 \times 200$ are used for training the model and for predictions.
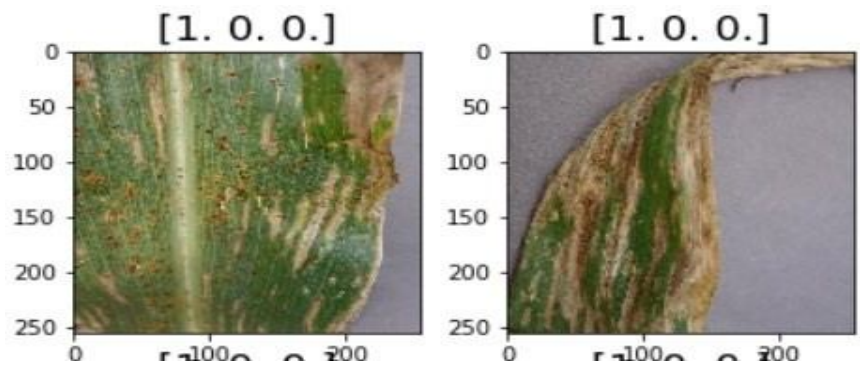

Figure 5: Pre-Processed Images After Labelling

\section{Model Development}

Model improvement includes showing a system of counterfeit of neurons, called neural networks with the picture information that is named as the info. At that point the neural system learns all the highlights present in the pictures and will have the option to order them dependent on those highlights. For the improvement of model for the Disease Detection Networks the contribution to the neural system is picture information that contains maize leaves with names. In the wake of learning, the neural system will have the option to foresee leaf ailment.

To accomplish this, a convolutional neural networks calculation is utilized which contains different layers and various capacities playing out some scientific procedure on the information and its marks gave. Convolutional neural network with all connected layers is shown in Fig 1.6.

The neural network that is used for training for the Disease Detection System contains about 260 thousand parameters and they continuously undergo changes as they learn from the images of dimensions $250 \times 200$.

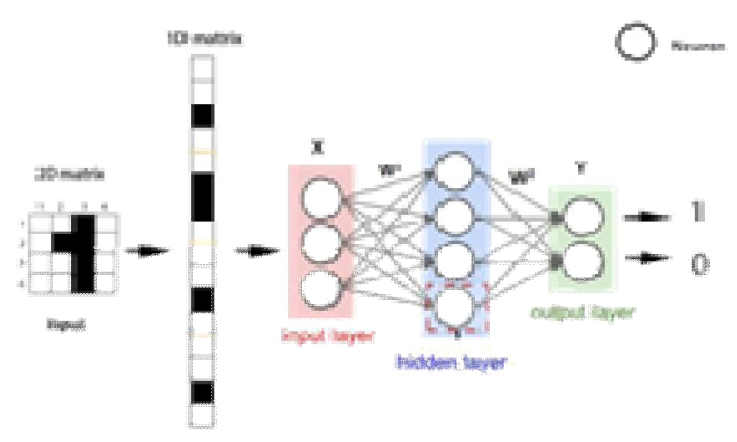

Figure $6: 2 \mathrm{D} \mathrm{CNN}$ 


\section{Defining the Neural network}

Defining the neural network involves adding all the functions to a network The neural network is defined with one input layer, three obscure layers and one result layer. The output activation function used is sigmoid, which generates the output in the form of 0's and 1's, as Disease Detection System assesses the leaves

\section{Supplying Data to Neural Network}

The data containing pre-processed images of leaves are supplied as the input to the neural network. The input data is stored in a directory and a data generator is created which helps in flowing the data from the directory to the neural network as it learns to classify. Then the neural network is trained, and a model is generated using the image data.

The model generated is saved into a file with extensions, json and h5. This model is loaded whenever prediction of an image is required. These files are stored in the server and is used whenever necessary

\section{RESULTS}

The results are shown in figure 7. The model is validated for training accuracy after completion of the training. The model is validated using 550 images and the validation accuracy is shown in figure 8 .
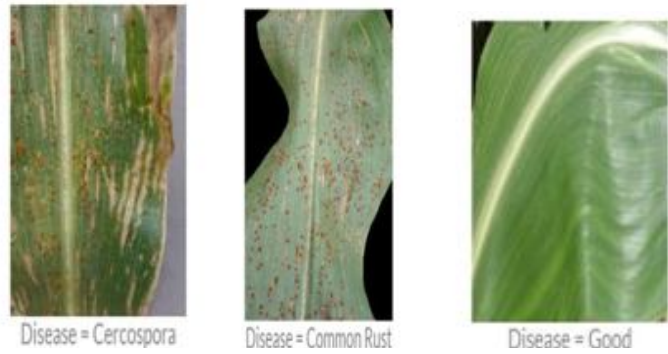

In [12]: modele evaluate generator(validation generator, no validation sampless)

Out[12]: $[0.048255995858418772,0.9959532374100719]$

Figure 8 : Training accuracy

Next the model is tested for overall accuracy at the completion of the development of the prototype. The accuracy of the prototype is shown in the figure 9 . The testing is done using 53 images of maize leaves

\section{loaded_model.evaluate_generator(test_generator, nb_test_samples)}

\section{$[1.150555618331308,0.8678886029422534]$}

Figure 9 :: Over all Model accuracy after testing

\section{Confusion Matrix}

A Confusion Matrix is an outline of probability outcomes on an order issue. The number of right and off base expectations are outlined with check values and divided by every group. This is the route towards the disarray grid. The perplexity framework indicates the methods through which your gathering prototype is confused when it makes forecasts. It provides us understanding not only interested in the blunders being made by a classifier but more crucially the types of mistakes which are made. The disarray network for the Disease Detection System is given underneath. The data that can be acquired from the perplexity grid is appeared in the Table 1. It contains data like exactness, review, f1-score, support and furthermore precision for that test information

Table 1 : Confusion Matrix

\begin{tabular}{|l|c|c|}
\hline Original & Positive & Negative \\
\hline Positive & 39 & 2 \\
\hline Negative & 4 & 8 \\
\hline
\end{tabular}

Precision: Precision is defined as the amount of accurate positives divided by the number of true positives plus the number of false positives.

Precision $=$ True positives $/$ (True positives + False Positives $)$

Recall: The precise definition of recall is the amount of true positives divided by the amount of true positives plus the amount of false negatives.

Recall $=$ True Positives $/$ (True Positives + False Negatives)

F1-score: The F1 score is the harmonic mean of precision and recall taking both metrics into account in the following equation:

$\mathrm{F} 1=2 *($ Precision $*$ Recall $) /($ Precision + Recall $)$

Support: The support is the number of occurrences of each class. The disease detection of leaves has been assessed by the model with an accuracy of $99.4 \%$. This model can be considered as a fairly good model because out of 50 good leaves, it has classified 49 leaves correct.

\section{CONCLUSION}

There are several methods in mechanized or PC image plant malady detection and characterization method, but at the similar point in time, this discovery ground is deficient. What's more, there are nevertheless no commercial preparations available, apart after individuals managing plant species acknowledgment dependent on the leaves pictures. Right now, new methodology of utilizing profound learning strategy was investigated so as to consequently arrange and identify plant ailments from leaf images. The created model had the option to recognize leaf nearness and recognize sound leaves and 13 unique ailments, which can be outwardly. 
analyzed. The total strategy was depicted, separately, from gathering the pictures utilized for preparing and approval to picture preprocessing and expansion lastly the procedure of preparing the deep CNN and fine-tuning. Different tests were acted so as to check the presentation of recently made model The model which is utilized to anticipate gives just two classes of leaves as yield, they are sound or ailing. With more information and complex neural system, if the model is prepared, the Disease Detection System may relate to more precision

\section{REFERENCES}

1. K. A. Garrett, S. P. Dendy, E. E. Frank, M. N. Rouse, and S. E. Travers, "Climate change effects on plant disease: genomes to ecosystems," Annual Review of Phytopathology, vol. 44, pp. 489- 509, 2006.

2. S.M. Coakley, H. Scherm, and S. Chakraborty, "Climate change and plant disease management," Annual Review of Phytopathology, vol. 37, no. 1, pp. 399-426, 1999. https://doi.org/10.1146/annurev.phyto.37.1.399

3. S. Chakraborty, A. V. Tiedemann, and P. S. Teng, "Climate change: potential impact on plant diseases," Environmental Pollution, vol. 108, no. 3, pp. 317-326, 2000. https://doi.org/10.1016/S0269-7491(99)00210-9

4. A. J. Tatem, D. J. Rogers, and S. I. Hay, "Global transport networks and infectious disease spread," Advances in Parasitology, vol. 62, pp. 293-343, 2006.

5. J. R. Rohr, T. R. Raffel, J.M. Romansic, H. McCallum, and P. J. Hudson, "Evaluating the links between climate, disease spread, and amphibian declines," Proceedings of the National Academy of Sciences of the United States of America, vol. 105, no. 45, pp. 17436-17441, 2008. https://doi.org/10.1073/pnas.0806368105

6. T. Van der Zwet, "Present worldwide distribution of fire blight," in Proceedings of the 9th International Workshop on Fire Blight, vol. 590, Napier, New Zealand, October 2001.

7. S. A. Miller, F. D. Beed, and C. L. Harmon, "Plant disease diagnostic capabilities and networks," Annual Review of Phytopathology, vol. 47, pp. 15-38, 2009.

8. M. B. Riley, M. R. Williamson, and O. Maloy, "Plant disease diagnosis. The Plant Health Instructor," 2002. https://doi.org/10.1094/PHI-I-2002-1021-01

9. J. G. Arnal Barbedo, "Digital image processing techniques for detecting, quantifying and classifying plant diseases," Springer-Plus, vol. 2, article 660, pp. $1-12,2013$.

10. H. Cartwright, Ed., Artificial Neural Networks, Humana Press, 2015. https://doi.org/10.1007/978-1-4939-2239-0

11. I. Steinwart and A. Christmann, Support Vector Machines, Springer Science \& BusinessMedia, New York, NY, USA, 2008.

12. S. Sankaran, A. Mishra, R. Ehsani, and C. Davis, "A review of advanced techniques for detecting plant diseases," Computers and Electronics in Agriculture, vol. 72, no. 1, pp. 1-13, 2010.
13. P. R. Reddy, S. N. Divya, and R. Vijayalakshmi, "Plant disease detection techniquetool-a theoretical approach," International Journal of Innovative Technology and Research, pp. 91-93, 2015.

14. A.-K. Mahlein, T. Rumpf, P. Welke et al., "Development of spectral indices for detecting and identifying plant diseases," Remote Sensing of Environment, vol. 128, pp. 21-30, 2013. https://doi.org/10.1016/j.rse.2012.09.019

15. W. Xiuqing, W. Haiyan, and Y. Shifeng, "Plant disease detection based on near-field acoustic holography," Transactions of the Chinese Society for Agricultural Machinery, vol. 2, article 43, 2014.

16. A.-K. Mahlein, E.-C. Oerke, U. Steiner, and H.-W. Dehne, "Recent advances in sensing plant diseases for precision crop protection," European Journal of Plant Pathology, vol. 133, no. 1, pp. 197-209, 2012. https://doi.org/10.1007/s10658-011-9878-z

17. T. R. Reed and J. M. H. Dubuf, "A review of recent texture segmentation and feature extraction techniques," CVGIP: Image Understanding, vol. 57, no. 3, pp. 359-372, 1993.

18. I. Lenz, H. Lee, and A. Saxena, "Deep learning for detecting robotic grasps," The International Journal of Robotics Research, vol. 34, no. 4-5, pp. 705-724, 2015.

19. B. Alipanahi, A. Delong, M. T. Weirauch, and B. J. Frey, "Predicting the sequence specificities of DNA- and RNA-binding proteins by deep learning," Nature Biotechnology, vol. 33, no. 8, pp. 831-838, 2015. https://doi.org/10.1038/nbt.3300

20. L. Zhang, G.-S. Xia, T.Wu, L. Lin, and X. C. Tai, "Deep learning for remote sensing image understanding," Journal of Sensors, vol. 2016, Article ID 7954154, 2 pages, 2016.

21. J. Arevalo, F. A. Gonzalez, R. Ramos-Pollan, J. L. Oliveira, and M. A. G. Lopez, "Convolutional neural networks for mammography mass lesion classification," in Proceedings of the $37^{\text {th }}$ Annual International Conference of the IEEE Engineering in Medicine and Biology Society (EMBC '15), pp. 797-800, August 2015. https://doi.org/10.1109/EMBC.2015.7318482

22. Prashanthi, V. \& Devika, P. \& Kanakala, Srinivas., Identification of opportunities for coding in a network. International Journal of Recent Technology and Engineering. 7. 140-144,2019

23. Prashanthi, V., D. Suresh Babu, and CV Guru Rao. "Network Coding aware Routing for Efficient Communication in Mobile Ad-hoc Networks." International Journal of Engineering \& Technology(UAE),Volume7,issue3 (2018): 1474-1481.

24. Kanakala, S., Ananthula, V. R., \& Vempaty, P. (2014). Energy-efficient cluster based routing protocol in mobile ad hoc networks using network coding. Journal of Computer Networks and Communications, 2014, 351020. https://doi.org/10.1155/2014/351020

25. K.Srinivas, A.Nagaraju, S.Ramachandram and G.Narsimha, (2010) "Performance Evaluation of Routing Protocols in Static and Dynamic Ad-hoc Networks based on Energy Consumption" Second Vaagdevi International 
Conference on Information Technology for Real World Problems.

https://doi.org/10.1109/VCON.2010.16

26. Lakshmi, K. Prasanna, and C. R. K. Reddy. "A survey on different trends in data streams." 2010 International Conference on Networking and Information Technology. IEEE, 2010.

27. Dhanalaxmi, B., G. Apparao Naidu, and K. Anuradha. "Adaptive PSO based association rule mining technique for software defect classification using ANN." Procedia Computer Science 46 (2015): 432-442. https://doi.org/10.1016/j.procs.2015.02.041

28. Prashanthi, V. \& Kanakala, Srinivas. (2018). Comprehensive data analysis of aarogyasri scheme progress in Telangana. Journal of Advanced Research in Dynamical and Control Systems. 10. 421-434.

29. V.Prashanthi, Srinivas Kanakala," Generating analytics from web $\log$ “,International Journal of Engineering and Advanced Technology (IJEAT)ISSN: 2249 -8958, Volume-9Issue-4, April 2020. https://doi.org/10.35940/ijeat.C6451.049420

30. P. Santhi , N. Deeban , N. Jeyapunitha , B. Muthukumaran and R. Ravikumar, Prediction of Diabetes using Neural Networks, International Journal of Advanced Trends in Computer Science and Engineering, Volume 9 No.2 (2020),pg.no.985-990.

31. KollaBhanu Prakash, Lakshmi Kalyani. N, Pradeep Kumar Vadla and Naga Pawan YVR, Analysis of Mammography for Identifying Cancer Cells using Convolution Neural Networks, International Journal of Advanced Trends in Computer Science and Engineering, Volume 9 No.2 (2020),pg.no-1184-1188. 